PAPER

\title{
Twenty four hour pulse pressure predicts long term recurrence in acute stroke patients
}

\author{
G Tsivgoulis, K Spengos, N Zakopoulos, E Manios, K Xinos, D Vassilopoulos, K N Vemmos
}

J Neurol Neurosurg Psychiatry 2005;76:1360-1365. doi: 10.1136/jnnp.2004.057265

See end of article for authors' affiliations

.....................

Correspondence to:

Dr K Spengos, Department of Neurology-University of Athens, Vasilissis Sofias 82, 11528 Athens, Greece; spengos@hol.gr

Received 27 October 2004 Revised version received 27 December 2004

Accepted 17 January 2005

\begin{abstract}
Objectives: The impact of different blood pressure (BP) components during the acute stage of stroke on the risk of recurrent stroke is controversial. The present study aimed to investigate by 24 hour BP monitoring a possible association between acute $\mathrm{BP}$ values and long term recurrence.

Methods: A total of 339 consecutive patients with first ever acute stroke underwent 24 hour BP monitoring within 24 hours of ictus. Known stroke risk factors and clinical findings on admission were documented. Patients given antihypertensive medication during BP monitoring were excluded. The outcome of interest during the one year follow up was recurrent stroke. The Cox proportional hazard model was used to analyse association of casual and 24 hour BP recordings with one year recurrence after adjusting for stroke risk factors, baseline clinical characteristics, and secondary prevention therapies.

Results: The cumulative one year recurrence rate was $9.2 \%$ (95\% Cl 5.9\% to $12.3 \%$ ). Multivariate Cox regression analyses revealed age, diabetes mellitus, and 24 hour pulse pressure (PP) as the only significant predictors for stroke recurrence. The relative risk for one year recurrence associated with every $10 \mathrm{~mm} \mathrm{Hg}$ increase in 24 hour PP was $1.323(95 \% \mathrm{Cl} 1.019$ to $1.718, \mathrm{p}=0.036)$. Higher casual PP levels were significantly related to an increased risk of one year recurrence on univariate analysis, but not in the multivariate Cox regression model.

Conclusions: Elevated 24 hour PP levels in patients with acute stroke are independently associated with higher risk of long term recurrence. Further research is required to investigate whether the risk of recurrent stroke can be reduced to a greater extent by decreasing the pulsatile component of BP in patients with acute stroke.
\end{abstract}

$\mathrm{T}$ he value of antihypertensive treatment in protection gainst first ever and recurrent stroke regardless of baseline blood pressure (BP) level is well established. ${ }^{12}$ In contrast, there is little consensus about optimal management of BP in the acute stroke setting, which is still an issue of debate. ${ }^{3}$ More specifically, the impact of the different components of BP during the acute stage of stroke on the risk of recurrent stroke is currently not clear. Data from observational studies have suggested that the BP values at the time of admission are related to early ${ }^{4-6}$ and late stroke recurrence, ${ }^{57}$ whereas other reports do not support such an association. ${ }^{89}$ Moreover, in a population based study neither the level of BP before the index event nor the acute BP values had any apparent effect on stroke recurrence throughout the follow up period. ${ }^{10}$

Compared with casually recorded BP, non-invasive 24 hour $\mathrm{BP}$ monitoring reduces the pressor response to hospital admission, ${ }^{11}$ diminishes the measurement variability and observer bias, ${ }^{12}$ and correlates more closely with hypertensive target organ damage. ${ }^{13}$ In addition, it provides a large number of readings that allows one to observe more precisely a rapidly evolving phenomenon-such as the BP course in the acute stage of stroke.

Therefore we aimed to investigate, by means of 24 hour BP monitoring, a possible association between $\mathrm{BP}$ values in the acute stage of stroke and the risk of long term recurrence.

\section{PATIENTS AND METHODS}

Data collection

Between January 1998 and January 2002, 415 consecutive patients with first ever stroke were admitted from the emergency room to the stroke unit of our hospital within 24 hours of the onset of symptoms. Nearly $90 \%$ of the patients with stroke in our stroke unit use the emergency services and are therefore admitted to hospital in the acute stage of stroke. The hospital is a university institution providing tertiary care services to the urban population of the city of Athens. All patients hospitalised after June 1992 have been included in a prospective observational stroke data bank. Details of the stroke patients in this registry from a five year period (June 1992-May 1997) have been previously described. ${ }^{14}$

For the present study, either an internist specialising in stroke or a neurologist examined the patients on admission and assessed stroke severity using the Scandinavian Stroke Scale. The Scandinavian Stroke Scale score ranges from 0 (minimum) to 58 (maximum), and the total score decreases with increasing stroke severity. ${ }^{15}$ All patients underwent brain computed tomography (CT) scanning and electrocardiography on admission, Doppler ultrasonography of the cervical arteries within 24 hours, and a second CT scan or magnetic resonance imaging (MRI) during the time they were in hospital. Selected patients (59\%) underwent transthoracic or transoesophageal echocardiography. According to the Trial of Org 10172 in Acute Stroke Treatment (TOAST) criteria, ${ }^{16}$ we classified ischaemic strokes on the basis of aetiopathogenetic mechanisms into the following groups:
- large artery atherosclerotic stroke (LAA)
- cardioembolic stroke (CE)
- small artery occlusion or lacunar stroke (LAC)
- infarct of undetermined cause (IUC).

Abbreviations: $\mathrm{BP}$, blood pressure; $\mathrm{CE}$, cardioembolic stroke; $\mathrm{DBP}$, diastolic blood pressure; LAA, large artery atherosclerotic stroke; LAC, small artery occlusion or lacunar stroke; $\mathrm{ICH}$, intracerebral haemorrhage; IUC, infarct of undetermined cause; MBP, mean blood pressure; PP, pulse pressure; SBP, systolic blood pressure 
Cardiovascular risk factors were documented in all cases Patients were considered as having a history of hypertension if they had evidence of systolic blood pressure (SBP) above $140 \mathrm{~mm} \mathrm{Hg}$, or diastolic blood pressure (DBP) above $90 \mathrm{~mm} \mathrm{Hg}$ at any time before or four weeks after stroke onset, or if they had received any antihypertensive medication prior to the index event. ${ }^{17}$ The definitions of cigarette smoking, diabetes mellitus, heart failure, coronary artery disease, and hypercholesterolaemia have been described elsewhere. $^{14}$

\section{BP monitoring and management}

On hospital admission, the same internist measured casual supine BP in both arms on three separate occasions at intervals of 15 minutes, using a standard mercury sphygmomanometer. The mean value was taken after ensuring that no difference in the BP between the two arms was greater than $10 \mathrm{~mm} \mathrm{Hg}$. We used the oscillometric Spacelab 90209 equipment (Spacelab Inc, Redmond, WA) to monitor the 24 hour BP. The monitoring equipment was applied in the study patients within 24 hours of the onset of stroke and was set to obtain automatic BP readings at 15 minute intervals. Any patient with fewer than three readings per hour at any time of the 24 hour period was excluded from the study. A total of between 72 and 96 pairs (three to four readings per hour; mean (SD) count of recordings 84.8 (3.9)) of SBP and DBP values per patient per 24 hours were entered into our statistical analysis program. SBP, DBP, mean BP (MBP), pulse pressure (PP), and heart rate recordings were automatically averaged and presented as mean values of the 24 hour BP monitoring. We checked the accuracy of the BP monitoring devices monthly by taking 10 automatic and 10 auscultatory BP readings simultaneously from the same arm via a Y-tube. In all instances values did not differ more than $5 \mathrm{~mm} \mathrm{Hg}$.

Our policy of BP treatment in the setting of acute stroke was based on the "European Stroke Initiative" (EUSI) recommendations for stroke management. ${ }^{18}$ We treated post-stroke hypertension in only those patients who had critically elevated BP levels (SBP >220 mm Hg, DBP $>120 \mathrm{~mm} \mathrm{Hg}$ ). Pre-stroke antihypertensive therapy was discontinued during the acute stage of the cerebrovascular event. None of the study patients was given thrombolytic therapy. Patients with post-stroke hypotension were not given vasopressors in order to achieve pharmacological elevation of BP.

Patients with a difference of greater than $10 \mathrm{~mm} \mathrm{Hg}$ between the casual BP in the two arms $(n=5)$ or with missing admission casual BP values $(n=9)$ were excluded from further evaluation. A further 23 patients had to be excluded because they had fewer than 72 pairs (three readings per hour) of SBP and DBP values per 24 hours. In addition, 26 patients were excluded because they received antihypertensive medication $(n=13)$, or succumbed to $(n=11)$ or had a recurrent stroke $(n=2)$ during the BP monitoring. Thus, a total of 352 stroke patients fulfilled all the abovementioned criteria and were prospectively studied. During the first seven days after the index event 10 patients died, and a recurrent cerebrovascular event was documented in four subjects. Repeat assessments of casual and 24 hour BP were made one week following acute stroke in the remaining 338 survivors. None of these patients had critically elevated $\mathrm{BP}$ levels in the first week of ictus. Consequently, in accordance with the EUSI recommendations, ${ }^{18}$ we did not initiate antihypertensive treatment during this period. BP medication was initiated in patients with a documented history of hypertension 15-20 days after the index event according to the World Health Organization guidelines for the management of hypertension. ${ }^{17}$

\section{Follow up}

Secondary stroke prevention therapies-lipid lowering, antiplatelet agents, and oral anticoagulants-were administered to selected patients in keeping with the EUSI guidelines. ${ }^{18} \mathrm{~A}$ study investigator and a trained nurse prospectively followed up all surviving patients $1,3,6$, and 12 months after the index event. The outcome events of interest were recurrent stroke and death. To determine the occurrence of recurrent ischaemic stroke or intracerebral haemorrhage (ICH) we evaluated all the available information obtained from death certificates, hospital records, physicians' notes in private practice, necropsy findings, and the patients' clinical presentation at the regular follow up assessments. Recurrent stroke was defined as a cerebrovascular event of sudden onset, lasting more than 24 hours, subsequent to the initial stroke, which clearly resulted in a new neurological deficit or an increase in an existing deficit. ${ }^{6}$ Visualisation of a new lesion on brain imaging, involving an anatomical site or vascular territory different from that of the index event, was mandatory to support the diagnosis of recurrent stroke during the first three weeks after stroke onset. This requirement intended to ensure that systemic causes of clinical deterioration after an initial stroke (hypoxia, hypotension, hyperglycaemia, infection) and worsening of symptoms because of progression of the initial stroke were not misclassified as a recurrent cerebrovascular event. ${ }^{19}$

The causes of death were divided into the following subgroups:

- neurological damage (for example, brain oedema, transtentorial herniation)

- infections

- cardiovascular causes (myocardial infarction, sudden death due to cardiac arrhythmia, acute pulmonary oedema or heart failure, pulmonary embolism)

- recurrent stroke

- other causes (for example, renal failure, cancer)

- unknown causes.

Patients lost to follow up $(n=13)$ were excluded from further evaluation.

\section{Statistical analyses}

Data are presented as mean (SD) or as percentages. We evaluated the changes in BP between the initial recordings on admission and the repeat assessments on day 7 with the paired $t$ test. We estimated the cumulative probability of survival and recurrence after one year from the index event with the Kaplan-Meier product-limit method. To evaluate which factors contribute to long term recurrence, we used Cox proportional hazards analyses. Those factors that contributed to the outcome in the initial univariate analyses at $\mathrm{p}$ values $<0.1$ (because of the risk of type II error due to low statistical power in such an analysis) were included in the multivariate model as candidate variables and then removed by backward stepwise selection procedure. In the final multivariate analyses, $\mathrm{p}<0.05$ was considered as statistically significant. To confirm the robustness of the multivariate models, we also performed all multivariate analyses using a forward selection procedure. In addition, to assess whether the relation of BP values during the acute stage of stroke with long term recurrence was independent of individual average "tonic" BP level, we repeated all analyses in the subgroup of patients who underwent 24 hour BP monitoring both on admission and on day 7 after stroke onset, after correcting for differences between the two serial BP measurements. Associations are presented as relative risk (RR) with corresponding 95\% confidence interval (CI). The $\mathrm{BP}$ variables retained as independent predictors of stroke 
recurrence in the final model were divided into tertiles (up to and including the 33rd and 67th centiles). Kaplan-Meier curves of groups stratified by BP variables are presented here; we used the log-rank method to compare recurrence free survival after stroke between BP tertiles. The Statistical Package for Social Science (SPSS Inc, version 10.0 for Windows) was used for statistical analyses.

\section{RESULTS}

The final study population fulfilling all of the abovementioned inclusion criteria consisted of 339 patients with first ever stroke who underwent BP monitoring on hospital admission (325 underwent a second BP monitoring at day 7 of ictus). Ischaemic stroke was diagnosed in 269 patients (79.4\%), and $70(20.6 \%)$ presented with stroke symptoms due to ICH. The distribution of ischaemic stroke subtypes was as follows: LAA $21.2 \%(\mathrm{n}=57)$, CE $31.6 \%(\mathrm{n}=85)$, LAC $26.8 \%(n=72)$ and IUC $20.4 \%(n=55)$. Demographic data and the risk factors for stroke risk are presented in table 1 .

We found statistically significant differences $(p<0.001)$ between the casual and 24 hour levels of SBP, DBP, MBP, and PP on hospital admission. Furthermore, in the stroke patients who underwent 24 hour BP monitoring both on admission and one week after the onset of stroke there was a significant fall $(p<0.001)$ in the levels of all BP components between the two recordings.

During the one year follow up period, we documented 89 deaths $(26.3 \%)$ and 27 recurrent cerebrovascular events $(8.0 \%$ ) (22 ischaemic strokes $3 \mathrm{ICH}$, and 2 unspecified type of recurrence because of lack of follow up brain imaging). One month case fatality and recurrence rate was $12.4 \%$ $(\mathrm{n}=42)$ and $2.1 \%(\mathrm{n}=7)$, respectively. The cumulative one year mortality and recurrence rate was $24.2 \%$ (95\% CI $19.7 \%$ to $28.8 \%$ ) and $9.2 \%$ (95\% CI $5.9 \%$ to $12.3 \%$ ), respectively. The main causes of death during the first month of ictus were neurological damage (50.0\%) and infections (21.4\%), whereas during the remaining follow up period deaths were mainly due to cardiovascular diseases $(27.7 \%)$ and recurrent strokes (23.4\%; table 2 ).

To identify independent predictors of long term recurrence we performed Cox proportional hazard analyses (table 3). In the initial univariate analyses age, diabetes mellitus, coronary artery disease, and casual and 24 hour PP were related to stroke recurrence and were therefore selected for the final multiple variable model. No statistically significant associations were identified between stroke recurrence and hypertension $(p=0.282)$, atrial fibrillation $(p=0.461)$, stroke subtype $(p=0.369)$, and stroke severity at hospital admission

Table 1 Demographics, risk factors and clinical presentation of 339 patients with acute first ever stroke

\begin{tabular}{ll}
\hline Age in years (mean (SD)) & $69.6(10.4)$ \\
Men (n (\%)) & $226(66.7)$ \\
Elapsed time (hours) from stroke onset to hospital & $6.1(6.9)$ \\
admission (mean (SD)) & \\
Hypertension (n (\%)) & $258(76.1)$ \\
Antihypertensive medication prior to the index & $176(51.9)$ \\
event (n (\%)) & \\
Diabetes mellitus (n (\%)) & $92(27.1)$ \\
Coronary artery disease (n (\%)) & $65(19.2)$ \\
Atrial fibrillation (n (\%)) & $93(27.4)$ \\
Heart failure (n (\%)) & $23(6.8)$ \\
Smoking (n (\%)) & $131(38.6)$ \\
Hypercholesterolaemia (n (\%)) & $91(26.8)$ \\
Previous transient ischaemic attack (n (\%)) & $27(8.0)$ \\
Scandinavian Stroke Scale score (mean (SD)) & $32.3(19.2)$ \\
Cerebral infarction (n (\%)) & $269(79.4)$ \\
Intracerebral haemorrhage (n (\%)) & $70(20.6)$ \\
\hline
\end{tabular}

Table 2 Causes of death during the first year after stroke

\begin{tabular}{lcc}
\hline Cause of death & $\leqslant$ month (\%) & $>$ 1 month (\%) \\
\hline Neurological damage & 50.0 & 4.3 \\
Infection & 21.4 & 17.0 \\
Cardiovascular diseases & 9.5 & 27.7 \\
Recurrent stroke & 11.9 & 23.4 \\
Other & 4.8 & 8.5 \\
Unknown & 2.4 & 19.1 \\
\hline
\end{tabular}

$(p=0.778)$. The multivariate Cox regression analyses (performed with both backward and forward selection procedures) revealed only increasing age, diabetes mellitus, and 24 hour PP as significantly independent outcome predictors. The relative risk for stroke recurrence associated with every $10 \mathrm{~mm} \mathrm{Hg}$ increase in 24 hour PP was 1.323 (95\% CI 1.019 to $1.718, \mathrm{p}=0.036)$. Since atrial fibrillation and history of hypertension are well documented risk factors for stroke recurrence, we repeated all analyses after incorporating these two variables in the multivariate Cox proportional hazards model and obtained similar results. Finally, the ischaemic stroke and the ICH subgroups were analysed separately; 24 hour PP was found to be an independent predictor of long term recurrence in the ischaemic stroke (RR 1.350, 95\% CI 1.038 to $1.755, \mathrm{p}=0.025$ ), but not in the ICH (RR $1.298,95 \%$ CI 0.854 to $1.911, \mathrm{p}=0.283$ ) population, due to the limited number of patients in this subgroup.

Cox regression analyses were also performed in the subgroup of patients $(\mathrm{n}=325)$ who underwent BP monitoring both on admission and one week after the onset of stroke. Interestingly, 24 hour PP levels on admission (RR 1.476, 95\% CI 1.133 to $1.922, p=0.004$ ) as well as at day 7 (RR 1.320 , $95 \%$ CI 1.027 to $1.696, p=0.030$ ) predicted long term recurrence on single variable analyses. However, only admission 24 hour PP values remained a poor prognostic factor (RR 1.340, 95\% CI 1.011 to $1.776, p=0.042$ ) in the multivariate model. In contrast, 24 hour PP recordings at day 7 were not independently $(p=0.161)$ associated with recurrent stroke in the final multiple regression analyses. Furthermore, increasing 24 hour PP levels were independently associated with one year mortality (RR 1.293, 95\% CI 1.106 to $1.512, p=0.001$ ) even after adjustment for baseline characteristics and secondary prevention therapies. KaplanMeier curves of patients stratified by admission 24 hour PP tertiles are presented in fig 1 . Subjects with 24 hour PP $\leqslant 59 \mathrm{~mm} \mathrm{Hg}$ (lowest tertile) had the lowest recurrence rate compared with the subgroup of patients with 24 hour PP of $>59 \mathrm{~mm} \mathrm{Hg}$ and $\leqslant 71 \mathrm{~mm} \mathrm{Hg}$ (median tertile) and those with 24 hour PP $>71 \mathrm{~mm} \mathrm{Hg}$ (higher tertile, log rank 6.76, $\mathrm{p}=0.034$ ). It is also interesting that in the first few weeks patients in the lowest PP tertile seemed to have the highest risk of stroke recurrence (see fig 1). It was not until after the first month that the patients with the highest PP levels started to deviate from the lowest tertile group and showed a more significant drop in recurrence free survival.

\section{DISCUSSION}

To our knowledge, this is the first study to evaluate the possible association between $\mathrm{BP}$ values in the acute stage of stroke and long term recurrence with the use of 24 hour BP monitoring. We documented a linear association between increasing 24 hour PP during the acute phase and recurrent stroke. This association retained statistical significance even after correcting for the average BP levels recorded one week following the index event, indicating that stroke recurrence cannot be solely attributed to post-stroke hypertension. 
Table 3 Results of the univariate and multivariate Cox proportional hazard analyses to determine the effect of different factors on recurrence

\begin{tabular}{|c|c|c|}
\hline Dependent variable & $\begin{array}{l}\text { Univariate analysis } \\
\text { Relative risk }(95 \% \mathrm{Cl})\end{array}$ & $\begin{array}{l}\text { Multivariate analysis } \\
\text { Relative risk }(95 \% \mathrm{Cl})\end{array}$ \\
\hline \multicolumn{3}{|l|}{ Baseline characteristics } \\
\hline Age (per 10 year increase) & $1.929(1.26$ to 2.953$) * * *$ & $1.980(1.256 \text { to } 3.121)^{* * *}$ \\
\hline Male sex & 0.924 (0.415 to 2.057$)$ & \\
\hline Hypertension & 1.791 (0.619 to 5.177$)$ & \\
\hline Diabetes mellitus & $2.180(1.020$ to 4.657$)$ ** & $2.342(1.072$ to 5.116$)$ ** \\
\hline Hypercholesterolaemia & $1.279(0.575$ to 2.847$)$ & \\
\hline Smoking & 1.161 (0.543 to 2.480 ) & \\
\hline Coronary heart disease & $1.998(0.874$ to 4.566$)$ * & 1.387 (0.583 to 3.303$)$ \\
\hline Atrial fibrillation & 0.694 (0.263 to 1.834$)$ & \\
\hline Heart failure & $1.961(0.590$ to 6.515$)$ & \\
\hline Previous transient ischaemic attack & $1.369(0.412$ to 4.548$)$ & \\
\hline SSS score (per 10 point increase) & 1.031 (0.834 to 1.275$)$ & \\
\hline \multicolumn{3}{|l|}{$\begin{array}{l}\text { Stroke subtype } \\
\text { Solnt }\end{array}$} \\
\hline Lacunar stroke & 1.0 & \\
\hline Large artery atherosclerotic stroke & $2.822(0.850$ to 9.375$)$ & \\
\hline Cardioembolic stroke & $2.363(0.666$ to 8.378$)$ & \\
\hline Infarct of undetermined cause & $1.653(0.466$ to 5.865$)$ & \\
\hline Intracerebral haemorrhage & 1.067 (0.239 to 4.773$)$ & \\
\hline \multicolumn{3}{|l|}{ Secondary prevention therapies } \\
\hline Diuretics & $0.854(0.400$ to 1.826$)$ & \\
\hline$\beta$-blocking agent & $1.866(0.817$ to 4.264$)$ & \\
\hline Calcium antagonist & $0.720(0.249$ to 2.085$)$ & \\
\hline Angiotensin-converting enzyme inhibitor & 0.631 (0.289 to 1.379$)$ & \\
\hline Angiotensin II receptor blockers & $0.284(0.038$ to 2.090$)$ & \\
\hline Lipid lowering agents & $1.037(0.438$ to 2.453$)$ & \\
\hline Antiplatelet agents & 0.641 (0.288 to 1.428$)$ & \\
\hline Oral anticoagulants & $0.658(0.228$ to 1.905$)$ & \\
\hline \multicolumn{3}{|l|}{ Blood pressure recordings } \\
\hline Casual SBP (per $10 \mathrm{~mm} \mathrm{Hg}$ increase) & $1.049(0.891$ to 1.234$)$ & \\
\hline Casual DBP (per $10 \mathrm{~mm} \mathrm{Hg}$ increase) & 0.865 (0.664 to 1.126$)$ & \\
\hline Casual MBP (per $10 \mathrm{~mm} \mathrm{Hg}$ increase) & $1.038(0.813$ to 1.325$)$ & \\
\hline Casual PP (per $10 \mathrm{~mm} \mathrm{Hg}$ increase) & 1.228 (0.981 to 1.537$)$ * & $0.878(0.622$ to 1.239$)$ \\
\hline 24 hour SBP (per $10 \mathrm{~mm} \mathrm{Hg}$ increase) & $1.094(0.910$ to 1.314$)$ & \\
\hline 24 hour DBP (per $10 \mathrm{~mm} \mathrm{Hg}$ increase) & $0.810(0.616$ to 1.065$)$ & \\
\hline 24 hour MBP (per $10 \mathrm{~mm} \mathrm{Hg}$ increase) & $0.951(0.748$ to 1.210$)$ & \\
\hline 24 hour PP (per $10 \mathrm{~mm} \mathrm{Hg}$ increase) & $1.455(1.138$ to 1.859$)$ ** & $1.323(1.019 \text { to } 1.718)^{* *}$ \\
\hline 24 hour heart rate (per $10 \mathrm{bpm}$ increase) & 0.867 (0.664 to 1.132$)$ & \\
\hline
\end{tabular}

${ }^{*} \mathrm{p}<0.1,{ }^{* *} \mathrm{p}<0.05,{ }^{* * *} \mathrm{p}<0.01$

DBP, diastolic blood pressure; MBP, mean arterial blood pressure; PP, pulse pressure; SBP, systolic blood pressure; SSS, Scandinavian Stroke Scale.

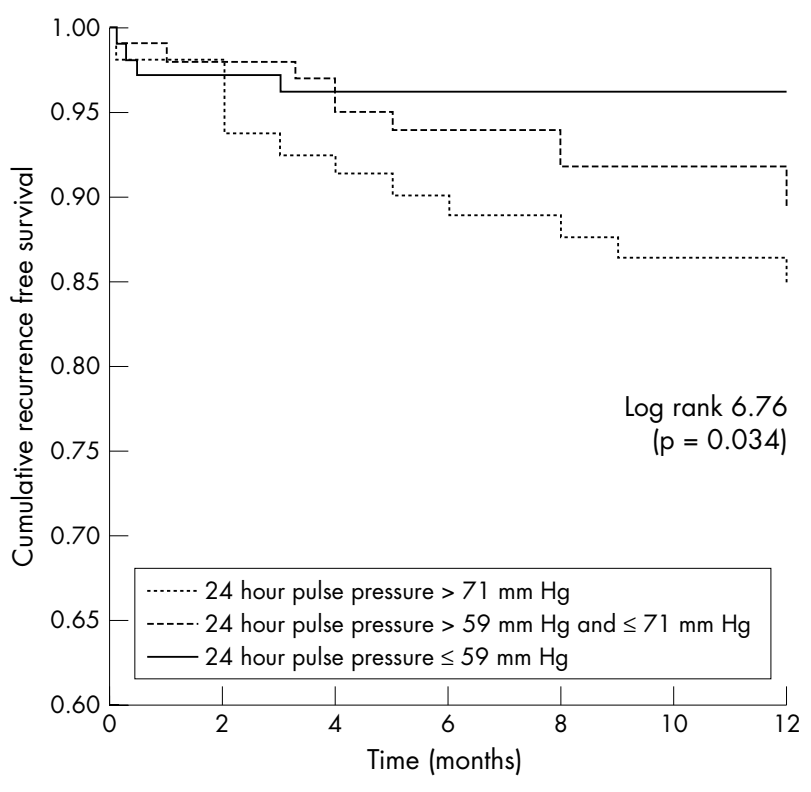

Figure 1 Kaplan-Meier curves of recurrence free survival after stroke in patients stratified into tertiles by admission 24 hour pulse pressure.
Previous studies have reported a relation between poststroke hypertension and stroke recurrence using casual BP measurements. ${ }^{4-10}$ However, the impact of different components of BP during the acute stage of stroke on early as well as on late recurrence is controversial. Elevated levels of both $\mathrm{SBP}^{47}$ and $\mathrm{DBP}^{67}$ on hospital admission have been shown to be related to early and late stroke recurrence. However, others have failed to document any association between admission BP values and recurrent stroke. ${ }^{8}{ }^{9}$ Moreover, in a population based study ${ }^{20}$ and a retrospective case series ${ }^{21}$ the BP levels recorded within one month of ictus had no apparent effect on the risk of recurrence.

The discrepancy between the results of the various studies may reflect underlying methodological problems such as BP levels measured by different observers, ${ }^{46-10} 2021$ retrospective BP documentation, ${ }^{10} 2021$ and lack of data on how BP was recorded. ${ }^{20}$ Another confounding factor is that the associations reported between BP and recurrent stroke were not assessed independently of other prognostic factors such as severity of stroke, ${ }^{702021}$ stroke subgroup, ${ }^{8102021}$ and concomitant risk factors. ${ }^{1021}$ Variation in the time of BP recording (ranging from $<2^{4}$ to $<30$ days $^{920}{ }^{21}$ ) may also account for some of the abovementioned conflicting results; the strategy of dichotomising BP, ${ }^{6792021}$ although clinically relevant might have created artificial strata for the analyses. ${ }^{3}$ Finally, DBP was not recorded in the International Stroke Trial, ${ }^{4}$ and thus the relation between other BP components (PP or MBP) and outcome could not be investigated. 
In the present study we initiated BP monitoring within 24 hours of ictus. None of the patients was given antihypertensive therapy during the recording. We carried out a thorough set of clinical and laboratory investigations to classify the patients into subtypes based on different aetiopathogenic mechanisms. The technique of non-invasive 24 hour BP monitoring, which is known to reduce measurement variability and observer bias, ${ }^{12}$ was used to overcome some of the methodological problems associated with BP measurement. Furthermore, casual and 24 hour BP values were forced in the regression model as continuous variables, avoiding dichotomisation and possible bias. We even investigated the association between acute $\mathrm{BP}$ values and risk of recurrent stroke after adjusting for BP levels at a different time point, when acute BP changes are no longer evident. Finally, all secondary stroke prevention therapies instituted in our cohort were included in the analysis as potential confounders.

The impact of PP on the risk of stroke seems to differ between study populations consisting of patients from different age groups. The incidence of stroke was better predicted by casual ${ }^{22}$ or 24 hour $\mathrm{MBP}^{23}$ values in middle aged patients with essential hypertension, whereas, in contrast, casual $^{24}$ and 24 hour $\mathrm{PP}^{25}$ values had a stronger association with risk of stroke in older people with isolated systolic hypertension in randomised clinical trials. A prospective study using intra-arterial ambulatory BP monitoring corroborated the abovementioned findings. The investigators reported that the relative significance of BP components depends on age: DBP measures provided the best prognostic value of stroke risk in middle aged individuals ( $<60$ years), whereas PP measures had the highest predictive power in the elderly group ( $\geqslant 60$ years). ${ }^{26}$ We would also like to point out that we have previously found an independent relation between elevated 24 hour PP in the acute stroke stage and one year mortality that was mainly due to recurrent stroke and cardiovascular events. ${ }^{27}$ Likewise, investigators in the Glycine Antagonist in Neuroprotection (GAIN) International Trial recently demonstrated that increased weighted average PP (documented by repeated casual recordings during the first hours of ictus) was the only BP component that was consistently associated with all four outcome measures of the study (mortality, handicap, disability, and severity of stroke)..$^{28}$

Elevated PP levels accelerate the progression of preclinical atherosclerosis, ${ }^{29}$ predict carotid artery stenosis, ${ }^{30}$ and are independently associated with carotid plaque ulceration. ${ }^{31}$ These structural changes in the large vessels are the underlying cause of LAA. PP has also been found to be related to increased stiffness of small vessels in animal models ${ }^{32}$ and in humans. ${ }^{33}$ Furthermore, resolution of small vessel remodelling was more closely related to changes in PP than in MBP. ${ }^{32}$ Increased prevalence and severity of white matter lesions, which are directly associated with lacunar stroke, correlate with higher PP values. ${ }^{34}$ In addition, elevated PP levels have been shown to be related to increased left atrial size, which in turn has been identified as a precursor of atrial fibrillation and stroke associated with atrial fibrillation. ${ }^{35}$ Finally, an association has been reported between PP at the time of presentation to hospital and ICH in conjunction with thrombolytic therapy for acute myocardial infarction. ${ }^{36}$

Our analysis was not intended to explain the possible mechanisms for the association of recurrent stroke with PP. However, it can be argued that in our study population, which comprised older patients with hypertensive acute stroke, elevated PP levels increased the risk of more advanced atherosclerosis and of plaque rupture in the large arteries supplying the brain. In addition, increased pulsatile stretching may further damage the small vessels of the cerebral circulation causing increased stiffness or rupture of cerebral arterioles.

Higher casual PP levels were related to an increased risk of one year recurrence on univariate analysis, but this association did not retain statistical significance in the multivariate Cox regression model. This finding may be attributed in the statistically significant difference between casual and 24 hour PP levels on hospital admission. In addition, the higher number of measurements, the absence of digit preference and observer bias, ${ }^{12}$ and minimisation of the white-coat effect $^{11}$ probably contributed to the predictive superiority of 24 hour over casual PP. The superiority of variables that describe the $\mathrm{BP}$ course in the acute stage of stroke in predicting stroke outcome when compared with single casual baseline measurements has been demonstrated by numerous reports, ${ }^{27} 2837$ whereas ambulatory BP variables have been shown to have stronger predictive power than screening BP measurements for stroke risk in a general population. $^{38}$

Our study had some limitations. Firstly, the body mass index of the patients was not recorded; thus this important vascular risk factor was not included in the analyses. Secondly, we defined a number of risk factors such as smoking and hypertension as binary variables although duration and severity are important. Thirdly, potential observer bias during follow up affecting the documentation of recurrent cerebrovascular events should be considered. It is of note though, that the recurrent strokes were mostly devastating or fatal and, in most instances, were confirmed by means of hospital records, brain imaging, and autopsy findings. Fourthly, there was potential risk of rebound hypertension and/or cardiac ischaemia in some stroke patients whose pre-stroke antihypertensive medications (especially $\beta$-blockers) were abruptly discontinued. Lastly, association does not meet causality and since this was an observational study, it cannot prove a causal relation of PP with stroke recurrence.

In conclusion, our results suggest that higher 24 hour PP values during the acute phase of stroke are independently associated with increased one year recurrence. This observation raises the possibility that the risk of recurrent stroke could be decreased to a greater extent by reducing the pulsatile component of BP in patients with acute stroke. Antihypertensive medications can selectively alter different components of BP and have been shown to have a differential effect on conduit vessel stiffness. This differential effect may translate into improved efficacy with agents that preferentially decrease large artery stiffness and therefore decrease PP values, such as angiotensin-converting enzyme inhibitors, nitric oxide donors, and low dose diuretics. However, before a case can be made for acute antihypertensive treatment, further research is needed to clarify the potential mechanisms (carotid plaque rupture, increased small vessel stiffness) that link acute elevation of PP with stroke recurrence independent of chronic hypertension.

\section{ACKNOWLEDGEMENTS}

The authors would like to thank Miss M Batsara for her valuable help in data collection.
Authors' affiliations
G Tsivgoulis, K Spengos, N Zakopoulos, E Manios, K Xinos,
D Vassilopoulos, K N Vemmos, Acute Stroke Unit of the Department of Clinical Therapeutics and Neurology, University of Athens Medical School, Athens, Greece
Competing interests: none declared 


\section{REFERENCES}

1 Chalmers J, Todd A, Chapman N, et al. International Society of Hypertension Writing Group. International Society of Hypertension (ISH): statement on blood pressure lowering and stroke prevention, J Hypertens 2003;21:651-63.

2 Donnan GA, Davis SM, Thrift A. The role of blood pressure lowering before and after stroke. Curr Opin Neurol 2003;16:81-6.

3 Willmot M, Leonardi-Bee J, Bath PM. High blood pressure in acute stroke and subsequent outcome: a systematic review. Hypertension 2004;43:18-24.

4 Leonardi-Bee J, Bath PM, Phillips SJ, et al. Blood pressure and clinical outcomes in the International Stroke Trial. Stroke 2002;33:1315-20.

5 Eriksson SE, Olsson JE. Survival and recurrent strokes in patients with different subtypes of stroke: a fourteen-year follow-up study. Cerebrovasc Dis 2001; 12:171-80.

6 Sacco RL, Foulkes MA, Mohr JP, et al. Determinants of early recurrence of cerebral infarction. The Stroke Data Bank. Stroke 1989:20:983-9.

7 Hier DB, Foulkes MA, Swiontoniowski M, et al. Stroke recurrence within 2 years after ischaemic infarction. Stroke 1991;22:155-61.

8 Wu TH, Chen HH, Lee TK. Factors affecting the first recurrence of noncardioembolic ischaemic stroke. Thromb Res 2000;97:95-103.

9 Moroney JT, Bagiella E, Paik MC, et al. Risk factors for early recurrence after ischaemic stroke: the role of stroke syndrome and subtype. Stroke 1998;29:2118-24.

10 Meissner I, Whisnant JP, Garraway WM. Hypertension management and stroke recurrence in a community (Rochester, Minnesota, 1950-1979). Stroke 1988; 19:459-63.

11 Fotherby MD, Critchley D, Potter JF. Effect of hospitalization on conventional and 24-hour blood pressure. Age Ageing 1995;24:25-9.

12 Coats AJ. Reproducibility or variability of casual and ambulatory blood pressure data: implications for clinical trials. J Hypertens Suppl 1990;8:S17-20.

13 Verdecchia P, Schillaci G, Guerrieri $M$, et al. Circadian blood pressure changes and left ventricular hypertrophy in essential hypertension. Circulation 1990;81:528-36

14 Vemmos KN, Takis CE, Georgilis K, et al. The Athens stroke registry: results of a five-year hospital-based study. Cerebrovasc Dis 2000;10:133-41.

15 Lindestrom E, Boysen G, Christiansen LW, et al. Reliability of Scandinavian Neurological Stroke Scale. Cerebrovasc Dis 1991;1:103-11.

16 Adams HP Jr, Bendixen BH, Kappelle $\amalg$, et al. Classification of subtype of acute ischaemic stroke. Definitions for use in a multicenter clinical trial. TOAST. Trial of Org 10172 in Acute Stroke Treatment. Stroke 1993;24:35-41

17 World Health Organization-International Society of Hypertension Guidelines for the Management of Hypertension. Guidelines Subcommittee. $J$ Hypertens 1999;17:151-83.

18 Hacke W, Kaste M, Skyhoi Olsen T, for the EUSI Executive Committee, et al Acute treatment of ischaemic stroke. European Stroke Initiative (EUSI). Cerebrovasc Dis 2000;10(suppl 3):22-33.

19 Toni D, Fiorelli M, Gentile M, et al. Progressing neurological deficit secondary to acute ischaemic stroke. A study on predictability, pathogenesis, and prognosis. Arch Neurol 1995;52:670-5.
20 Burn J, Dennis M, Bamford J, et al. Long-term risk of recurrent stroke after a first-ever stroke. The Oxfordshire Community Stroke Project. Stroke 1994;25:333-7.

21 Irie K, Yamaguchi T, Minematsu K, et al. The J-curve phenomenon in stroke recurrence. Stroke 1993;24:1844-9.

22 Millar JA, Lever AF, Burke V. Pulse pressure as a risk factor for cardiovascular events in the MRC Mild Hypertension Trial. J Hypertens 1999;17:1065-72.

23 Verdecchia P, Schillaci G, Reboldi G, et al. Different prognostic impact of 24 hour mean blood pressure and pulse pressure on stroke and coronary artery disease in essential hypertension. Circulation 2001;103:2579-84.

24 Domanski MJ, Davis BR, Pfeffer MA, et al. Isolated systolic hypertension: prognostic information provided by pulse pressure. Hypertension 1999;34:3753-80.

25 Staessen JA, Thiij L, O'Brien ET, et al. Ambulatory pulse pressure as predictor of outcome in older patients with systolic hypertension. Am J Hypertens 2002; 15:835-43.

26 Khattar RS, Swales JD, Dore C, et al. Effect of aging on the prognostic significance of ambulatory systolic, diastolic, and pulse pressure in essential hypertension. Circulation 2001;104:783-9.

27 Vemmos KN, Tsivgoulis G, Spengos K, et al. Pulse pressure in acute stroke is an independent predictor of long-term mortality. Cerebrovasc Dis 2004; 18:30-6.

28 Aslanyan S, Weir CJ, Lees KR; GAIN International Steering Committee and Investigators. Elevated pulse pressure during the acute period of ischaemic stroke is associated with poor stroke outcome. Stroke 2004;35:e153-5.

29 Lakka TA, Salonen R, Kaplan GA, et al. Blood pressure and the progression of carotid atherosclerosis in middle-aged men. Hypertension 1999;34:51-6.

30 Franklin SS, Sutton-Tyrrell K, Belle SH, et al. The importance of pulsatile components of hypertension in predicting carotid stenosis in older adults. J Hypertens 1997; 15:1143-50

31 Lovett JK, Howard SC, Rothwell PM. Pulse pressure is independently associated with carotid plaque ulceration. J Hypertens 2003;21:1669-76.

32 Baumbach GL, Siems JE, Heistad DD. Effects of local reduction in pressure on distensibility and composition of cerebral arterioles. Circ Res $1991 ; 68: 338-51$

33 James MA, Watt PA, Potter JF, et al. Pulse pressure and resistance artery structure in the elderly. Hypertension 1995;26:301-6.

34 Liao D, Cooper L, Cai J, et al. The prevalence and severity of white matter lesions, their relationship with age, ethnicity, gender, and cardiovascular disease risk factors: the ARIC Study. Neuroepidemiology 1997;16:149-62.

35 Vaziri SM, Larson MG, Lauer MS, et al. Influence of blood pressure on left atrial size. The Framingham Heart Study. Hypertension 1995;25:1155-60.

36 Selker HP, Beshansky JR, Schmid CH, et al. Presenting pulse pressure predicts thrombolytic therapy-related intracranial haemorrhage. Thrombolytic Predictive Instrument (TPI) Project results. Circulation 1994;90:1657-61.

37 Robinson TG, Dawson SL, Ahmed U, et al. Twenty-four hour systolic blood pressure predicts long-term mortality following acute stroke. J Hypertens $2001 ; 19: 2127-34$.

38 Ohkubo T, Hozawa A, Nagai K, et al. Prediction of stroke by ambulatory blood pressure monitoring versus screening blood pressure measurements in a general population: the Ohasama study. J Hypertens 2000;18:847-54. 